\title{
Activin receptor-like kinase 1 is essential for placental vascular development in mice
}

\author{
Kwon-Ho Hong, Tsugio Seki and S Paul Oh
}

Activin receptor-like kinase 1 (ALK1) is involved in the pathogenesis of hereditary hemorrhagic telangiectasia type II (HHT2) and pulmonary arterial hypertension. We have previously shown that Alk1 is predominantly expressed in the arterial endothelium and plays a pivotal role in the formation of embryonic blood vessels. At present, however, little is known about the precise expression pattern and function of ALK1 during extra-embryonic vascular development. Using previously generated lacZ reporter lines, we sought to examine the expression pattern and role of Alk1 during placental development in mice. Alk1 expression was restricted to endothelial cells of fetal vessels from the emergence of chorioallantoic fusion to the late gestational period, and no detectable Alk1 expression was observed in syncytiotrophoblasts or spongiotrophoblasts. Predominant arterial expression was observed in the umbilical and fetal placental vessels as well as in embryonic vessels. Morphological analysis of Alk1-null embryos indicates that Alk1 is essential for the development of distinct umbilical arteries and veins. The invasion of chorioallantoic mesoderm into the forming labyrinth layer was largely unaffected in the Alk1-null placenta, but chorioallantoic vessels appeared to be severely dilated and fused. Results from this study provide valuable information regarding the role of ALK1 in the development of placental vasculature as well as insights into the pathogenesis of $\mathrm{HHT}$.

Laboratory Investigation (2007) 87, 670-679; doi:10.1038/labinvest.3700560; published online 28 May 2007

KEYWORDS: ALK1; ALK5; TGF- $\beta$; blood vessel; placenta; HHT

Hereditary hemorrhagic telangiectasia (HHT) is an autosomal dominant vascular disorder occurring in one out of 10000 people. ${ }^{1,2}$ The most common clinical symptoms of HHT are nosebleeds and mucocutaneous telangiectases. Telangiectasic lesions exhibit dilation of the vascular lumen, thinning of the vascular wall, and arteriovenous malformations (AVMs) - direct connections between arterioles and venules without intervening capillaries. Large AVMs in the lungs, brain, liver, and gastrointestinal track of HHT patients can cause life-threatening complications. Genetic studies have revealed that heterozygous mutations in ENDOGLIN $(E N G)$ or Activin receptor-like kinase 1 (ALK1; ACVRL1) cause HHT1 and HHT2, respectively. ${ }^{3,4}$ Recent reports have shown that some SMAD4 mutations can also cause HHT. ${ }^{5}$

ENG and ALK1 are, respectively, a type III and type I receptor for transforming growth factor- $\beta$ (TGF- $\beta$ ) superfamily ligands. SMAD4 is a common mediator of all SMADdependent TGF- $\beta$ family signaling. It is conceivable that HHT is caused by impairment of mediating a TGF- $\beta$ family ligand common to ENG, ALK1, and SMAD4. Although TGF- $\beta 1$ and $\beta 3$ have been considered the most likely ligands for ENG/ALK1 signaling, as both ENG and ALK1 interact with multiple TGF- $\beta$ family ligands, the issue concerning the identity of the HHT-causing ligand for ENG/ALK1 remains unresolved. ${ }^{6}$

Heterozygous Alk1-null mice develop an array of HHT-like vascular malformations. ${ }^{7}$ As the frequency, time of onset, and location of vascular lesions in these mice are as unpredictable as in humans with HHT, the heterozygous mutants can serve as an animal model for HHT in studying additional genetic or environmental factors that affect the formation of vascular abnormalities. Homozygous Alk1-null mice die at midgestation around embryonic day (E) 10.5 with severe vascular defects including AVMs, impairment in the recruitment or differentiation of vascular smooth muscle cells (SMCs), and the hyperdilation/fusion of blood vessels. ${ }^{8,9}$ Although HHT is caused by a haploinsufficient condition of ALK $1,{ }^{10}$ the vascular malformations observed in homozygous Alk1-null mice are fundamentally the same as those in HHT patients. Thus, Alk1-null embryos could provide valuable information for

Department of Physiology and Functional Genomics, University of Florida College of Medicine, Shands Cancer Center, Gainesville, FL, USA Correspondence: Dr SP Oh, PhD, Department of Physiology and Functional Genomics, University of Florida College of Medicine, Shands Cancer Center, 1376 Mowry Road, Room 456, Gainesville, FL 32610, USA. E-mail: ohp@phys.med.ufl.edu 
determining the underlying molecular mechanisms for vascular malformations in HHT patients. As the onset of the Alk1-null phenotype coincides with the establishment of fetoplacental circulation, which is essential for the growth and vitality of embryos, we considered whether the phenotype of Alk1-null embryos is influenced by defects in placental development.

We have previously shown that detectable Alk1 expression was limited in the arterial endothelium during the embryonic and early postnatal stages. ${ }^{11}$ We also demonstrated that a $9.2 \mathrm{~kb}$ genomic fragment containing a $2.7 \mathrm{~kb}$ promoter region and the entire intron 2 of the Alkl gene is sufficient to drive arterial endothelium-predominant expression in transgenic mice. ${ }^{12}$ The differential Alk1 expression pattern contributes to the wealth of vascular heterogeneity, an emerging area of interest in vascular biology. Recent studies have revealed that specification of arterial and venous endothelial cells (EC) is determined at the level of angioblasts before the onset of circulation. Ephrin-B2 (Efnb2) was expressed in arterial EC, whereas Ephb4 was expressed in venous EC. ${ }^{13,14}$ Notch-activation by Notch ligands (eg Jag1 and Delta4) induces Efnb2 for arterial EC specification through downstream Gridlock/ Hey transcription factors, ${ }^{15,16}$ whereas Notch-suppression by an orphan nuclear receptor, COUP-TFII, specifies venous EC identity. ${ }^{17}$ It was reported that Efnb2 expression was suppressed in Alk1-null embryos, indicating that ALK1 may also specify arterial ECs at the early stage. ${ }^{9}$ In contradiction to this view, however, a recent study showed no functional interaction between Notch and ALK1 signalings. ${ }^{18}$ Our previous study implicated shear stress in inducing Alk1 expression in arterial ECs. ${ }^{11}$ Differences in oxygen tension between arteries and veins might be another factor that influences the differential Alk1 expression pattern. Studying Alk1 expression patterns in umbilical and placental vessels may provide additional clues regarding these matters.

As a maternal and fetal interface, the placenta is a vital organ for the exchange of gases, nutrients, and waste products. ${ }^{19,20}$ Morphogenesis of the chorioallantoic placenta begins with formation of the chorion from the extra-embryonic ectoderm and of the allantois from the mesoderm at the posterior end of the embryo during gastrulation. Subsequently, the allantois reaches the chorionic plate, and fetoplacental blood vessels are established by E8.5. Extensive branching morphogenesis by the allantoic mesoderm and the chorionic trophoblasts forms the labyrinth (Lab) layer, where maternal-fetal exchange occurs.

To investigate the function of ALK1 in placental vascular development, we examined expression patterns of Alk1 in umbilical and placental vessels as well as histological analyses of Alk1-null embryos. We demonstrate that heterogeneous Alk1 expression in vascular endothelium is conserved in extra-embryonic vasculature and is restricted to the fetal EC of placentae. We also show that Alk1 deficiency results in severe dilation and fusion of chorioallantoic and umbilical vesselsessentially the same as the embryonic phenotype-suggesting that the vasodilatory phenotype of the Alk1-null embryos is due primarily to Alk1 deficiency per se rather than to a secondary response to hypoxia. These data provide useful information for the future use of Alk1-null embryos as an animal model for studying the pathogenesis of HHT.

\section{MATERIALS AND METHODS}

All animal procedures performed were reviewed and approved by the University of Florida Institutional Animal Care and Use Committee. C57BL/6J and FVB wild-type mice were purchased from Harlan (Indianapolis, IN, USA).

\section{Mice}

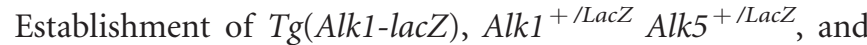
$F l k^{+/ L a c Z}$ reporter lines was described previously. ${ }^{11,12,21,22}$ Female $T g(A l k 1-l a c Z), A l k 1^{+/ L a c Z} A l k 5^{+/ L a c Z}$, and Flk1 ${ }^{+/ L a c Z}$ mice were intercrossed with wild-type males. The pregnant females were sacrificed at specific gestational days, and embryos and uteri/placentae were examined for lacZ expression patterns of maternal and fetal blood vessels in the placental and umbilical vessels. To generate Alk1-null mice, male and female $A l k 1^{+/ L a c Z}$ mice were intercrossed.

\section{X-gal Staining}

The harvested uterus/placenta samples were stained with Xgal as described previously. ${ }^{11}$ The uterine walls and yolk sacs were opened, and the placentae, with embryos attached, were immersed for $15 \mathrm{~min}$ in the fixative solution. At stages E12.5E15.5, the placentae were sagittally dissected in $1-2 \mathrm{~mm}$ slices for better penetration. After washing with PBS, the fixed uteri/placentae were stained with X-gal solution overnight. The stained samples were subsequently fixed with $4 \%$ paraformaldehyde and either embedded in paraffin for histology or cleared with organic solvent (Bezyl alcohol:Benzyl benzoate $=1: 1$; Sigma) for whole-mount imaging. The embedded samples were cut into $6-7 \mu \mathrm{m}$ slices.

\section{Histology and Immunohistochemistry}

To determine the X-gal-positive cells, the sections were counterstained with nuclear fast red (NFR) or immunostained with anti- $\alpha$ SMA (clone: 1A4; Sigma, 1:800), anti-platelet endothelial cell adhesion molecule (PECAM) (clone: Mec13.3; PharMingen, 1:200), or anti-keratin (Z0622, rabbit pAb; DAKO, 1:1200) antibodies. The standard ABC method was used with a Vector MOM staining kit (Vector laboratories Inc., CA, USA). After the secondary antibody reaction, color development was carried out with $\mathrm{DAB}^{+}$substrate chromogenic solution (Vector laboratories Inc., CA, USA).

\section{RESULTS}

Alk1 is Expressed in the Primitive Blood Vessels during Early Chorioallantoic Placental Development

Using previously generated $A l k 1^{+/ \operatorname{lacZ}}$ and $\operatorname{Tg}(A l k 1-\operatorname{lacZ})$ reporter lines, ${ }^{12,22}$ we investigated the expression pattern of Alk1 during early chorioallantoic placental development. The 
first detectable Alk1 expression was observed in the allantoic bud at around E7.75 (Figure 1a). At the subsequent stages, when the allantoic mesoderm forms primitive umbilical vessels and reaches to the chorionic plate, Alk1 expression was detected in these primitive vascular beds formed in the allantoic mesoderms as well as in the embryonic blood vessels (Figure 1b). Most X-gal-positive cells in these extra-embryonic tissues were positive for PECAM-1, an EC-specific marker (Figure 1c and d). Alk1 expression was apparent in patent blood vessels formed in the chorioallantoic connection of E9.0 embryos (Figure 1e and f).

\section{Alk1 is Differentially Expressed in the Umbilical Arterial Endothelium and Required for the Development of a Separate Umbilical Artery and Vein}

To further investigate whether the arterial endothelium-predominant expression pattern that we had observed in the embryo proper was conserved in extra-embryonic vessels, we examined the $\mathrm{X}$-gal staining pattern in the umbilical vessels of E15.5 Alk1 $1^{+/ l a c Z}$ and $\operatorname{Tg}(A l k 1$-lacZ) mice and compared it with that of $F l k 1^{+/ \text {lacZ }}$ and Alk5 $5^{+/ \text {lacZ }}$ mice. ${ }^{21,22} \mathrm{X}$-gal-positive cells were found mostly in the umbilical arterial endothelium of Alk1 ${ }^{+/ l a c Z}$ mice (Figure 2a and b). No X-gal-positive cells were found in vascular smooth muscle or adventitial layers, and the venous EC had very weak X-gal-positive staining. $\operatorname{Tg}($ Alk1-lacZ) mice exhibited the same expression pattern as the $A l k 1^{+/ l a c Z}$ mice, except that the X-gal staining intensity in the venous endothelium was stronger than that of $A l k 1^{+/ l a c Z}$ mice (Figure $2 \mathrm{c}$ and $\mathrm{d}$ ). In contrast, $\mathrm{X}$-gal-positive cells were found in both arterial and venous endothelia of umbilical vessels in $\mathrm{Flk1}^{+ \text {/lacZ }}$ mice (Figure 2e and f). Interestingly, the $\mathrm{X}$-gal-staining intensity in the venous endothelium was stronger than that in the arterial endothelium (Figure 2e). In addition, X-gal-positive staining in the microvessels surrounding medial layer of arteries were detected in $F l k 1^{+/ l a c Z}$ (Figure 2e), but not in $A l k 1^{+/ l a c Z}$ mice (Figure 2c). These results demonstrate heterogeneity of expression of endothelialspecific genes in a vessel-type-dependent manner. The Activin receptor-like kinase 5 (Alk5) expression was detected mostly in the medial layers but not in the endothelium of umbilical arteries or veins (Figure $2 \mathrm{~g}$ and $\mathrm{h}$ ). The non-overlapping expression patterns of these TGF- $\beta$ type I receptors are con-
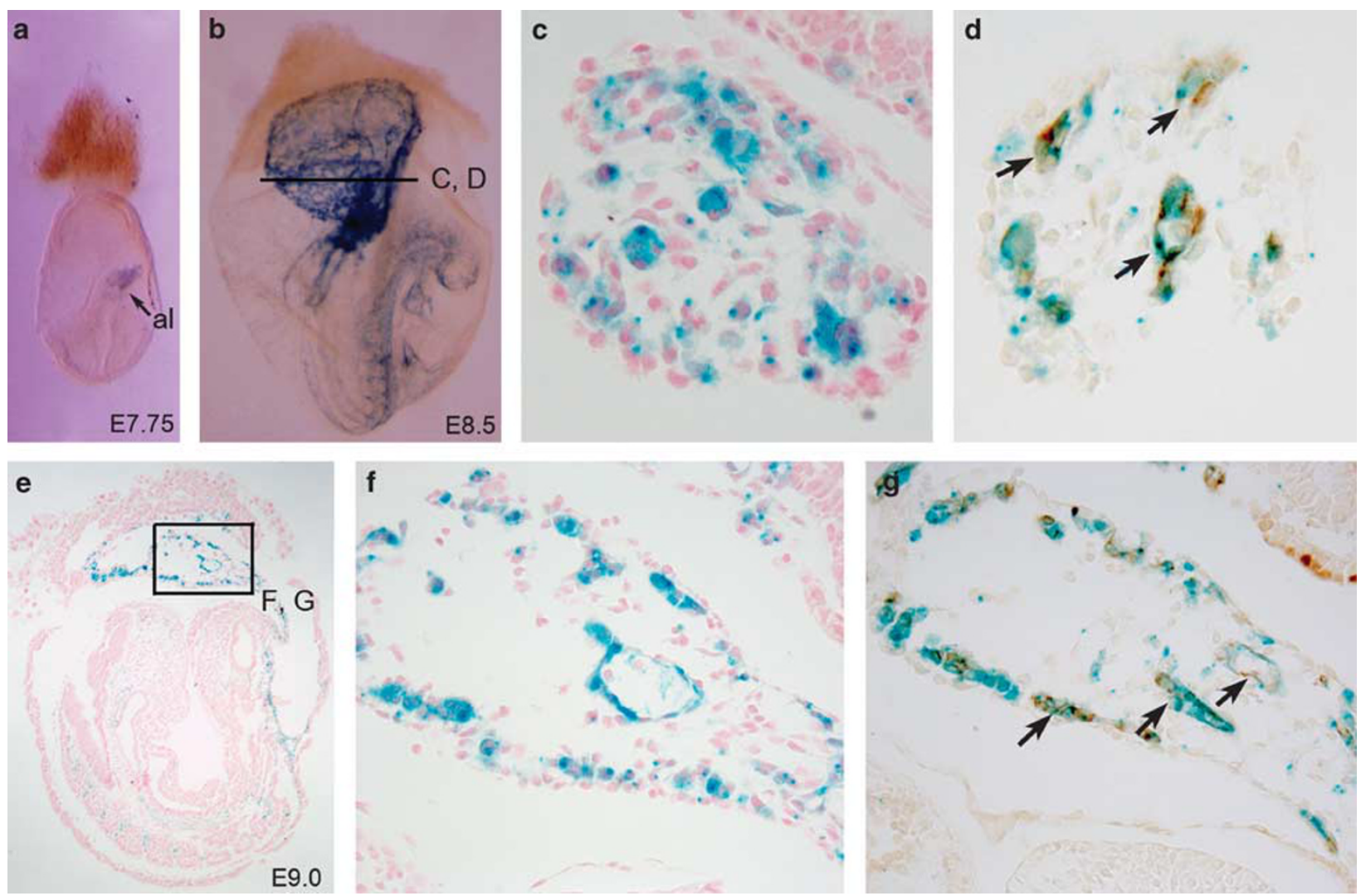

Figure 1 Whole-mount X-gal staining of Tg(Alk1-lacZ) embryos at days E7.75-E9.0 during early chorioallantoic development. (a) The first detectable Alk1 expression was found in the allantoic bud of E7.75 embryos. al, allantois. (b-d) X-gal staining was detected in the developing blood vessels of the embryo proper as well as in the primitive chorioallantoic vessels of E8.5 embryos. Transverse sections of X-gal stained E8.5 embryos at the level of the allantois were counterstained with NFR (c) or immunostained with anti-PECAM1 antibodies (d), showing that the majority of X-gal-positive cells are PECAM1-positive. $(\mathbf{e}-\mathbf{g})$ Longitudinal section of E9.0 embryos shows X-gal staining in embryonic and extra-embryonic ECs. (f, $\mathbf{g}$ ) Higher magnification views of allantoic mesoderm, showing development of patent blood vessels positive for X-gal and PECAM1. Arrows in (d) and (g) indicate some representative cells positive for both X-gal and PECAM1. 


\section{$\mathrm{X}-\mathrm{Gal} / \mathrm{NFR}$}
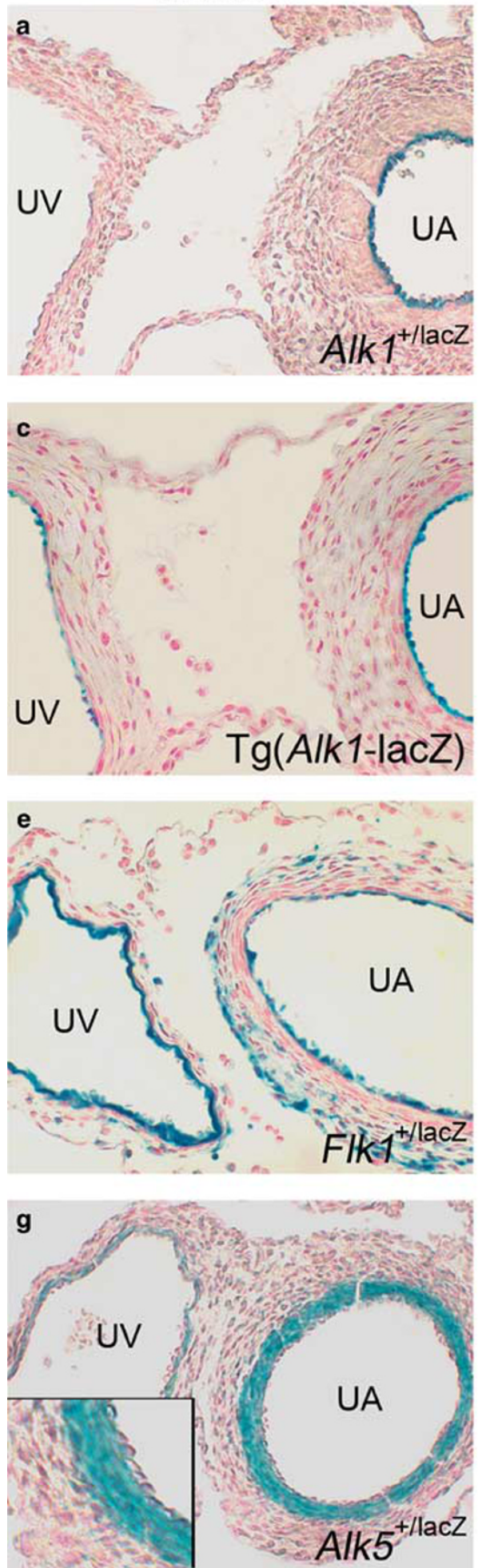

\section{X-Gal/ $\alpha$ SMA}
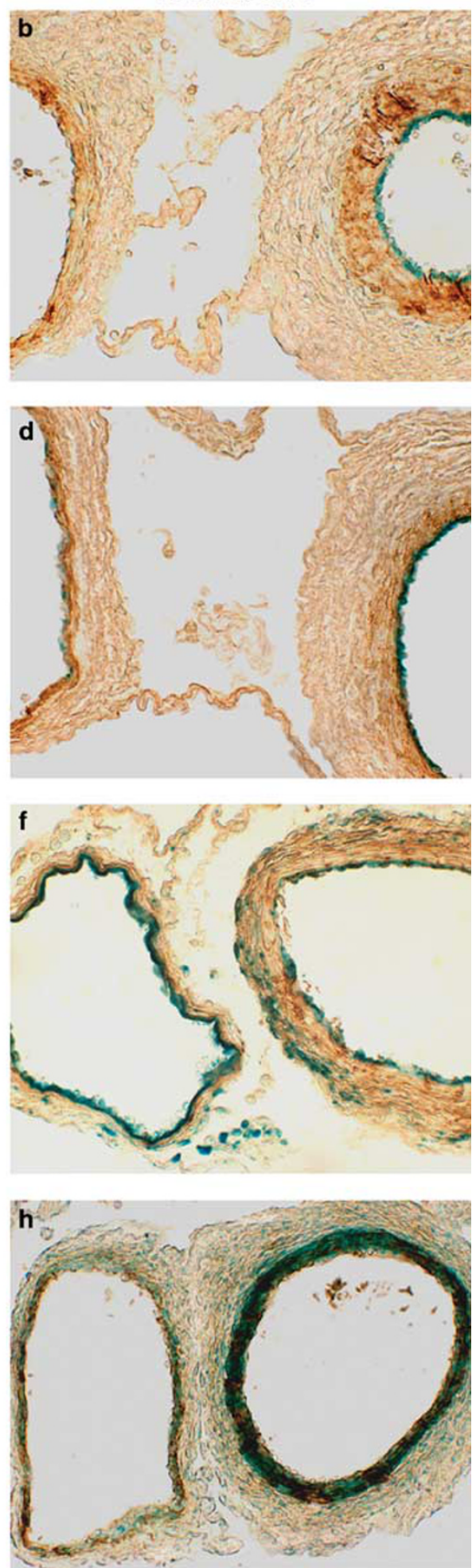

Figure 2 Expression patterns of Alk1, Flk1, and Alk5 in E15.5 umbilical vessels. Transverse sections of whole-mount X-gal-stained umbilical vessels of $A l k 1^{+/ l a c Z}(\mathbf{a}, \mathbf{b}), \operatorname{Tg}(A l k 1-\operatorname{lacZ})(\mathbf{c}, \mathbf{d}), F l k 1^{+/ \operatorname{lacZ}}(\mathbf{e}, \mathbf{f})$, and $A / k 5^{+/ l a c Z}(\mathbf{g}, \mathbf{h})$ embryos were counterstained with NFR $(\mathbf{a}, \mathbf{c}, \mathbf{e}, \mathbf{g})$ or anti- $\alpha \mathrm{SMA}$ antibodies $(\mathbf{b}, \mathbf{d}, \mathbf{f}, \mathbf{h})$, Note that X-gal-positive cells were mostly detected in the endothelium of the umbilical artery (UA) of Alk $1^{+/ l a c Z}$ (a, b) and Tg(Alk1-lacZ) embryos (c, d). X-gal-staining intensity was stronger in the umbilical vein (UV) than in the UA, and capillaries in the medial and adventitial areas were also X-gal-positive in Flk $1^{+ \text {llacZ }}$ mice $(\mathbf{e}, \mathbf{f})$. X-gal-positive cells were localized in smooth muscle layers, but not in endothelium of the UA and UV in Alk5 ${ }^{+/ l a c z}(\mathbf{g}, \mathbf{h})$. A highmagnification view of the UA is shown as an inset in $(\mathbf{g})$. 
sistent with the results of our previous study on embryonic vessels, suggesting that TGF- $\beta$ might be involved in vascular morphogenesis utilizing two distinct type I receptors: ALK1 in ECs and ALK5 in vascular SMCs.

The vessel-type-dependent differential expression patterns as well as the function of $A L K 1$ were investigated with $A l k 1^{+/ l a c Z}$ and Alk $1^{\text {lacZ/lacZ }}$ embryos at E9.5 and E10.5. The differential expression pattern was not obvious at the E9.5 stage (Figure 3a), but became noticeable by the E10.5 stage (Figure 3c). Umbilical arterial and venous lumens were clearly separated in Alk1 ${ }^{+/ l a c Z}$ mice along the entire length of umbilical vessels (Figure $3 \mathrm{a}$ and $\mathrm{c}$ ), whereas only a single large lumen was found in the Alk1-null embryos at both E9.5 (four out of four embryos) and E10.5 (three out of three embryos), especially around the middle of umbilical vessels (Figure $3 \mathrm{~b}$ and $\mathrm{d}$ ).

\section{Alk1 is Expressed in the Arteries and Capillaries in the Lab Layer of the Placenta}

Next, we examined Alk1 expression in the placentae during the mid- and late gestational stages. Expression pat- terns in both $\operatorname{Tg}\left(A l k 1\right.$-lacZ) and $A l k 1^{+/ l a c Z}$ placentae were essentially identical, although the overall staining intensity appeared to be higher in the $\mathrm{Tg}$ line (Figure $4 \mathrm{a}-\mathrm{f}$ ). The murine placenta is a multiple-layered organ consisting of endometrium-derived deciduas (Dec), junctional zones containing trophoblast giant cells, spongiotrophoblasts (Sp), and Lab where physiological exchanges occur. ${ }^{20}$ The Lab layer contains multiple cellular barriers: a single layer of mononucleated cytotrophoblasts, two layers of multinucleated syncytiotrophoblasts, and fetal capillary EC. ${ }^{19}$ Predominant arterial staining was again manifested, and fetal arteries projecting into the Lab layer of the definitive placentae were highlighted by the $\mathrm{X}$-gal staining. On the other hand, no $\mathrm{X}$-gal staining was detected in the $\mathrm{Sp}$ or Dec layers of the placentae in the Alk1-reporter lines. Dissimilar from Alk1reporter lines, X-gal-positive fetal arteries projecting into the Lab layer were unobservable in the $F l k 1^{+/ l a c Z}$ mice (Figure $4 \mathrm{~g}-\mathrm{i})$. In addition, Flk1 expression was detected in maternal vessels in the decidua and the uterine walls (Figure $4 \mathrm{~g}$ ).

Histological sections of X-gal stained Alk1 $1^{+ \text {lacZ }}$ placentae confirmed the restricted Alk1 expressions in the umbilical
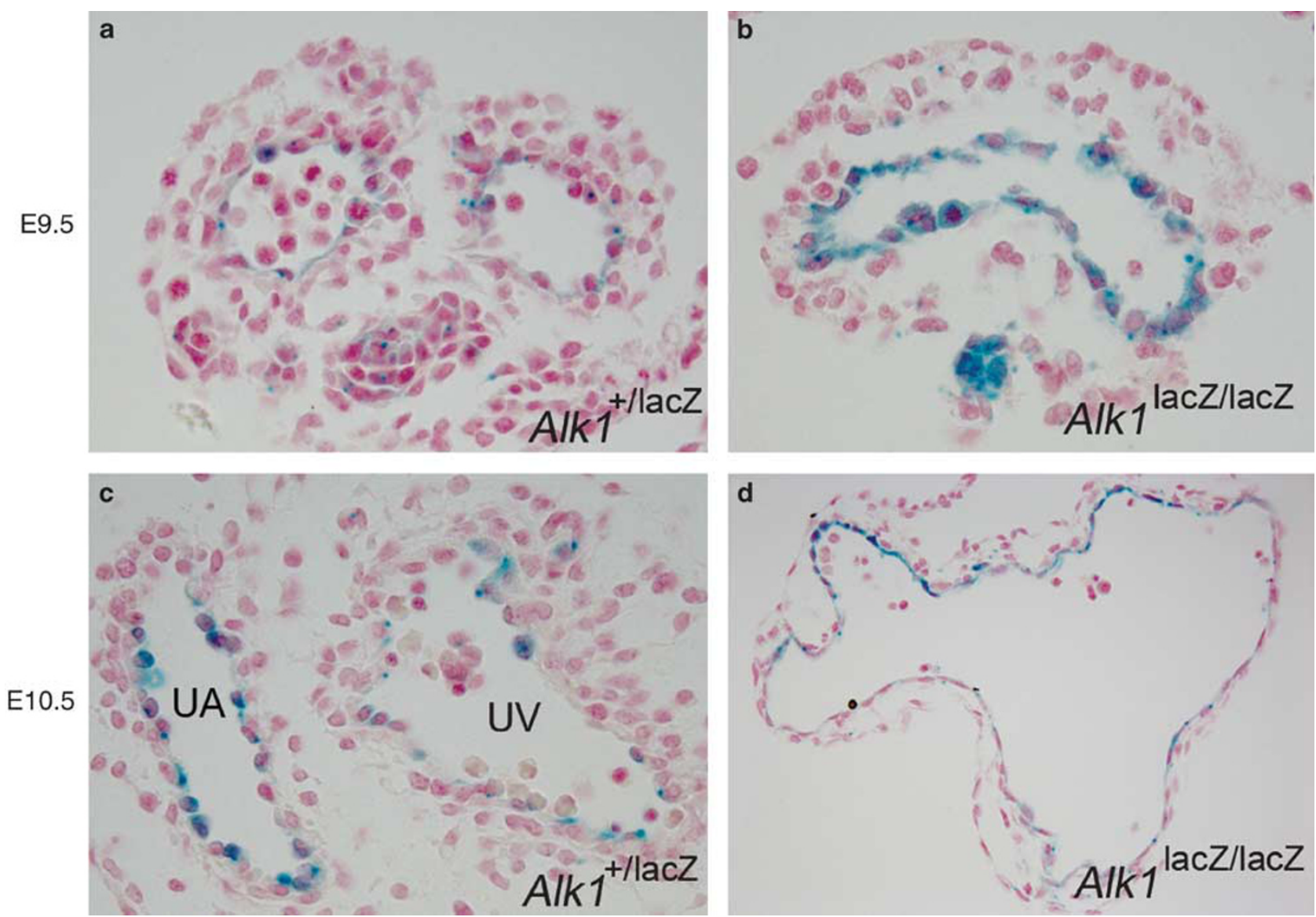

Figure 3 Impaired formation of two distinct umbilical vessels in Alk1-null embryos. Histological analysis of umbilical vessels of $A / k 1^{+/ l a c z}(\mathbf{a}, \mathbf{c})$ and Alk $1^{\text {lacZ/lacZ }}(\mathbf{b}, \mathbf{d})$ embryos at E9.5 $(\mathbf{a}, \mathbf{b})$ and E10.5 (c, d). Transverse sections of whole-mount X-gal-stained umbilical vessels were counterstained with NFR. Two distinct blood vessels were apparent in $A l k 1^{+/ \text {lacz }}$, whereas only one large persistent vessel was present in $A / k 1^{\text {lacz/lacz }}$ embryos. It is interesting to note that the UA-predominant Alk1 expression became evident in E10.5 embryos (c). 

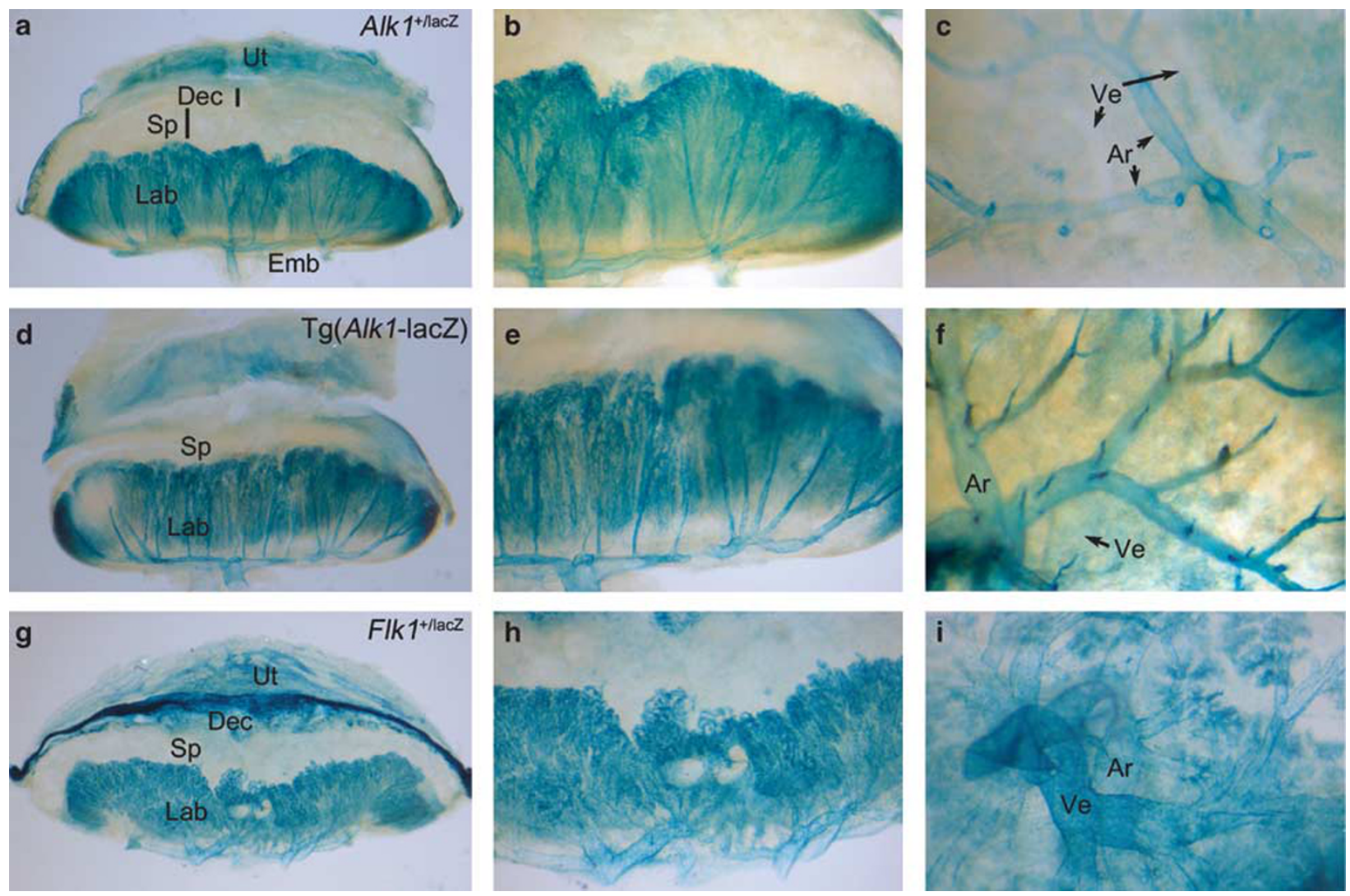

Figure 4 Comparison of Alk1 and Flk1 expression patterns in the definitive placentae. Whole-mount X-gal-stained placentae of E15.5 Alk1+/lacZ (a-c), $\operatorname{Tg}\left(\right.$ Alk1-lacZ) (d-f), and Flk1 $1^{+/ l a c Z}(\mathbf{g}-\mathbf{i})$ embryos were cleared with organic solvents. Higher magnification of longitudinal view of (a, d, $\left.\mathbf{g}\right)$ is presented in (b, e, h). (c, f, i) Horizontal view of X-gal-stained placenta from fetus side. Note that Alk1 expression is limited to the Lab layer and strong in fetal arteries projecting into the Lab layer. Ar, fetal arteries; Dec, decidua basalis; Emb, embryonic side; Lab, labyrinth layer; Sp, spongiotrophoblast layer; Ut, uterine wall; Ve, fetal veins.

vessels and the Lab layer (Figure 5a-d). No detectable X-gal staining was observed in fetal and maternal vessels in the $\mathrm{Sp}$ and Dec layers. To determine the cell type expressing Alk1 in the Lab layer, we stained E12.5 placenta sections with antiPECAM or cytokeratin (CK) antibodies. We used E12.5 placentae because the villus density in the Lab layer is less compact than that of E15.5, and thus different cell layers can be identified more easily. The X-gal-positive cells in the Lab layer appeared to be PECAM-positive but mostly non-overlapping with CK-positive cells, showing that Alk1 is expressed in EC but not in syncytiotrophoblasts.

\section{Alk1 Deficiency Results in Impairment of Umbilical and Placental Blood-Vessel Formation}

To investigate the role of Alk1 in placental development, we examined Alk1-null placentae at the E9.5 stage. The branching morphogenesis of the chorionic ectoderm and the invasion of allantoic mesoderm into the Lab layer of Alk $1^{\text {lacZ/lacZ }}$ placentae were largely unaffected (Figure 6a and b). In Alk1-null placentae, however, the chorionic vessels were severely dilated and showed signs of fusions, similar to the vascular abnormality observed in Alk1-null embryos. ${ }^{8}$

\section{DISCUSSION}

Using lacZ reporter lines, we showed heterogeneous Alk1 expression patterns in umbilical and fetal placental blood vessels and demonstrated that Alk1 deficiency leads to persistent umbilical vessels and extensive dilation of chorioallantoic vessels. Alk1 expression was first detected in the allantoic bud and then in the chorioallantoic primitive vessels when the allantois reaches the chorion. Alk1-null embryos formed a large persistent vessel instead of a separate umbilical artery and vein. At present, it is not clear whether this was due to the lateral fusion of the two umbilical vessels after their formation or to an absence of septation during their development.

At later stages, Alk1 was expressed predominantly in the arterial endothelium of umbilical vessels (Figure 2a), consistent with our previous findings in embryonic vessels. ${ }^{11}$ It is interesting to note that the vessel type-dependent differential Alk1 expression pattern becomes apparent at around E10.5 (Figure 3a and c). This is a relatively late stage in comparison with the vessel type-specific expression patterns of NotchDelta pathway genes and Efnb2/Ephb4 genes. Taking into account our previous data implicating shear stress as an 

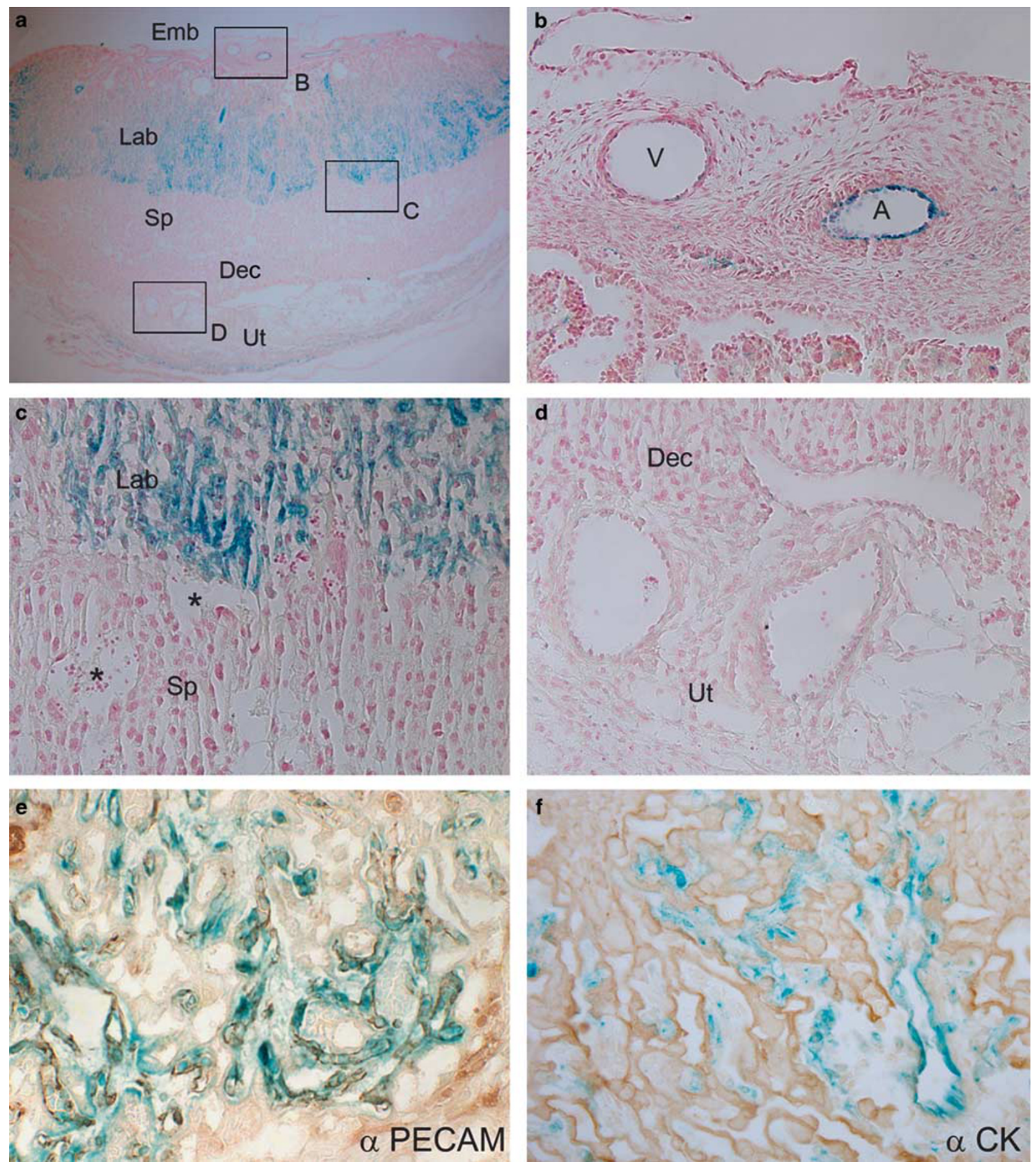

Figure 5 Longitudinal sections of X-gal-stained Alk1+/lacZ placenta. (a-d) Histological sections of E15.5 placenta shows that X-gal-positive cells are localized mostly in fetal blood vessels at umbilical arteries (b) and capillaries in the Lab layer (c), whereas no X-gal-positive cells are detected in the Sp (c) and Dec layers or in the uterine wall (d). (e, f) Immunostaining (shown as brown color) of the Lab areas of X-gal-stained E12.5 placenta with anti-PECAM1 (e) or antiCK (f) antibodies, showing that EC, but not syncytiotrophoblasts, are X-gal positive.

inducing mechanism for Alk1 expression, ${ }^{11}$ we speculate that the predominantly arterial Alk1 expression pattern could be influenced primarily by physiological differences—-such as hemodynamics-between two vessel types rather than ge- netic programming in angioblasts. Furthermore, the oxygen tension does not appear to be the underlying basis of the vessel-type-specific differential Alk1 expression, as the umbilical vessels-where the oxygen tension is reversed in 

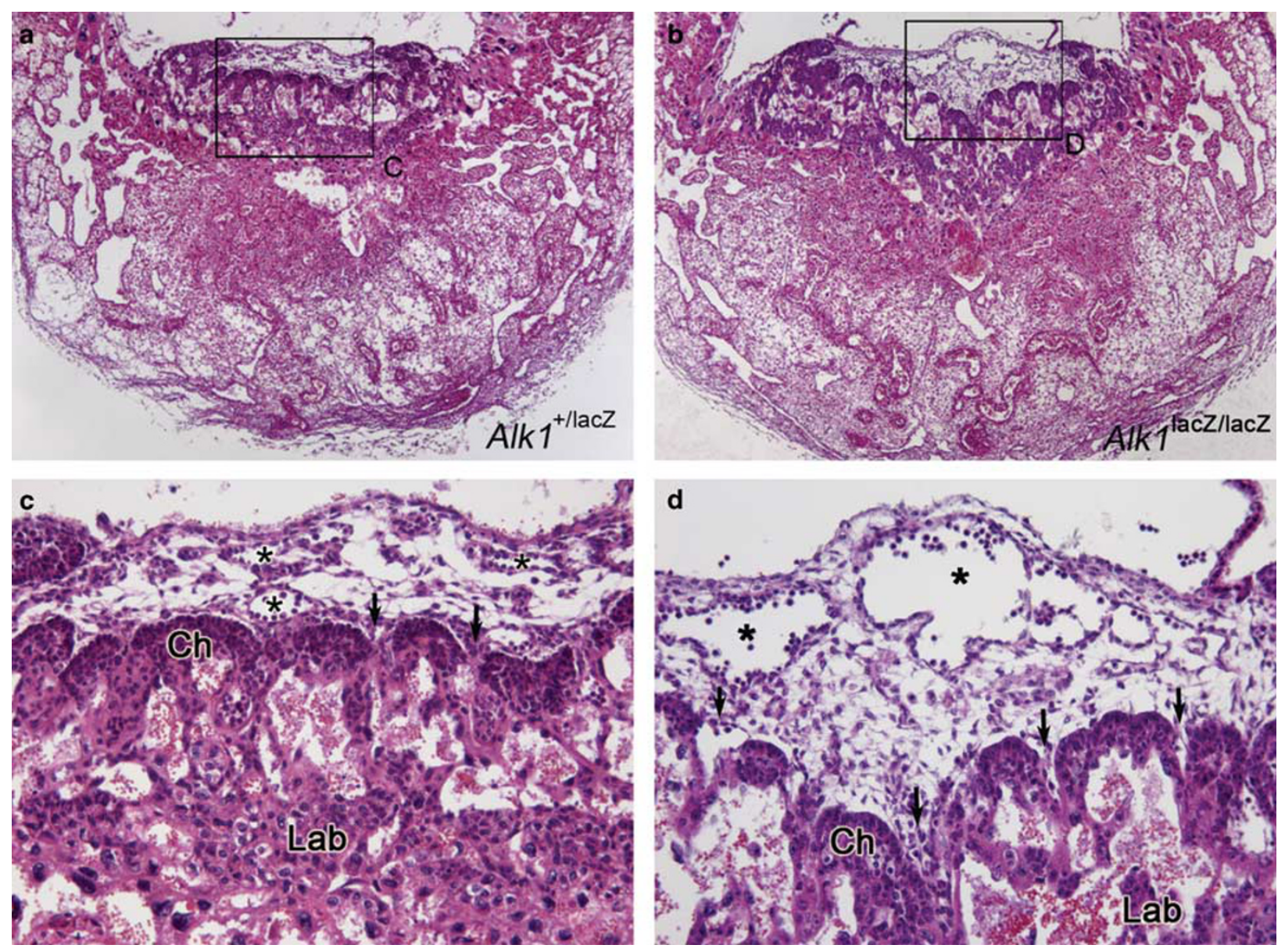

Figure $6 \mathrm{H} \& \mathrm{E}$ staining of histological sections of E9.5 Alk1 $1^{+/ l a c z}(\mathbf{a}, \mathbf{c})$ and $A l k 1^{\text {lacZ/lacz }}(\mathbf{b}, \mathbf{d})$ decidua. Asterisks and arrows in $\mathbf{c}$ and $\mathbf{d}$ indicate chorioallantoic blood vessels and areas of allantoic mesodermal invasion into developing Lab, respectively. Ch, chorionic ectodermal cells.

comparison with the embryonic vessels-displayed the expression pattern similar to that in embryonic vessels.

Alk1-null embryos die at around E10.5 with severe dilation and excessive fusion of blood vessels and also exhibit an enlarged pericardial space and markedly reduced trabeculation in the primitive ventricles. ${ }^{8}$ Although the severity of dilation in Alk1-null embryos is exceptional, the lethality at E10.5 with thin cardiac defects is a common phenotype of numerous other knockout embryos. Among genes involved in TGF- $\beta$ family signaling, targeted disruption of Alk1, Alk5 (Tgfbr1), Tgfbr2, Smad1, Smad5, Eng, or Tak1 (Map3k7) led to embryonic lethality at around E10.5. ${ }^{23-28}$ The study with mitogen-activated protein kinase 14 (Mapk14; $p 38 \alpha$ )knockout mice clearly demonstrated that impaired feto-placental circulation alone can lead to embryonic lethality at around E10.5 with cardiac defects. ${ }^{29}$ Therefore, careful characterization of placental development is crucial for the correct interpretation of embryonic phenotypes and the use of the mutant embryos for follow-up studies.

We demonstrated that the process of establishing the labyrinthine layer was unaffected in Alk1-null placentae: allantoic mesodermal cells were able to invade the chorionic plate and coalesced with trophoblasts to form the primitive Lab layer. The chorioallantoic vessels invading the Lab layer were, however, severely dilated to the same degree as the embryonic vessels. The dilation of chorionic vessels and the fusion of umbilical vessels certainly would affect feto-placental circulation and contribute to lethality and the cardiac phenotype in Alk1-null embryos. However, the vascular malformations observed in extra-embryonic vessels are essentially identical to those of the Alk1-null embryo proper, suggesting that the phenomenon of blood-vessel dilation/ fusion is due primarily to Alk1 deficiency rather than to a secondary physiological response to impaired feto-placental circulation. Indeed, several mutant mice with placental defects and embryonic lethality did not exhibit dilation of chorioallantoic vessels. For example, both Alk5- and Flt1 (Fms-like tyrosine kinase)-knockout embryos had defects in the formation of the Lab layer: there was no invasion of allantoic mesoderms into the chorionic plate, yet no dilation or fusion of chorioallantoic vessels was observed. ${ }^{24,30}$

Preeclampsia is characterized by hypertension and proteinuria in the third trimester of pregnancy and occurs in 5\% of all pregnancies. ${ }^{31,32}$ Deficiency of trophoblast invasion and 
spiral artery remodeling leads to increased uteroplacental vascular resistance and poor placentation, and results in stimulation of producing 'toxic' factors by the placenta. Recent studies have shown that an elevated level of soluble FLT1 (sFLT1) and sENG of placental origin may contribute to this malady. ${ }^{33,34}$ Unlike Flk1, whose expression is limited to EC, strong fetal Flt expression was observed in Sp and syncytiotrophoblasts in addition to EC in mice. ${ }^{30}$ Eng was also shown to be highly expressed in the syncytiotrophoblasts in human placentae. $^{35}$

ALK1 and ENG are plasma-membrane proteins that mediate TGF- $\beta$ family signals, and both are involved in the same genetic disease and are expressed in vascular EC. Because of these common characteristics between ENG and ALK1, the question emerges of whether ALK1 is also involved in preeclampsia. It must be if the pathogenetic mechanisms of preeclampsia involve the inhibition of a TGF- $\beta$ family signal mediated by ENG and ALK1. However, several studies have suggested that ENG function may not be limited to mediating TGF- $\beta$ family signals. ${ }^{36,37}$ Non-overlapping expression patterns between Alk1 and Eng also substantiate this possibility that ENG functions independently of ALK1. We showed that the placental expression pattern of Alk1 was different from that of Eng or Flt1: Alk1 expression was restricted to fetal EC, whereas Eng and Flt1 were expressed in both fetal endothelial and placental trophoblasts. ${ }^{30,35}$ In the sinusoidal EC of the liver, Eng expression was strong, whereas Alk1 expression was undetectable. ${ }^{11,38}$ In addition, the arterial-EC-predominant expression of Eng was not as apparent as that of Alk1. ${ }^{38}$

The data presented in this paper provide useful information for utilizing Alk1-lacZ reporter lines and Alk1-null embryos in the investigation of placental vascular development as well as of pathogenetic mechanisms for HHT and preeclampsia.

\section{ACKNOWLEDGEMENTS}

We thank Kirk Conrad for critical reading of the paper, and Marya Park for editorial assistance. This work was supported by NIH (HL64024) and AHA (0455336B) to SPO, and by AHA predoctoral fellowship to $\mathrm{K}-\mathrm{HH}$.

1. Guttmacher $A E$, Marchuk DA, White Jr RI. Hereditary hemorrhagic telangiectasia. N Engl J Med 1995;333:918-924.

2. Abdalla SA, Letarte M. Hereditary haemorrhagic telangiectasia: current views on genetics and mechanisms of disease. J Med Genet 2006;43:97-110.

3. McAllister KA, Grogg KM, Johnson DW, et al. Endoglin, a TGF-beta binding protein of endothelial cells, is the gene for hereditary haemorrhagic telangiectasia type 1. Nat Genet 1994;8:345-351.

4. Johnson DW, Berg JN, Baldwin MA, et al. Mutations in the activin receptor-like kinase 1 gene in hereditary haemorrhagic telangiectasia type 2. Nat Genet 1996;13:189-195.

5. Gallione CJ, Richards JA, Letteboer TG, et al. SMAD4 mutations found in unselected HHT patients. J Med Genet 2006;43:793-797.

6. David L, Mallet C, Mazerbourg S, et al. Identification of BMP9 and BMP10 as functional activators of the orphan activin receptor-like kinase 1 (ALK1) endothelial cells. Blood 2007;109:1953-1961.
7. Srinivasan S, Hanes MA, Dickens T, et al. A mouse model for hereditary hemorrhagic telangiectasia (HHT) type 2. Hum Mol Genet 2003;12:473-482.

8. Oh SP, Seki T, Goss KA, et al. Activin receptor-like kinase 1 modulates transforming growth factor-beta 1 signaling in the regulation of angiogenesis. Proc Natl Acad Sci USA 2000;97:2626-2631.

9. Urness LD, Sorensen LK, Li DY. Arteriovenous malformations in mice lacking activin receptor-like kinase-1. Nat Genet 2000;26:328-331.

10. Abdalla SA, Pece-Barbara N, Vera S, et al. Analysis of ALK-1 and endoglin in newborns from families with hereditary hemorrhagic telangiectasia type 2. Hum Mol Genet 2000;9:1227-1237.

11. Seki T, Yun J, Oh SP. Arterial endothelium-specific activin receptor-like kinase 1 expression suggests its role in arterialization and vascular remodeling. Circ Res 2003;93:682-689.

12. Seki T, Hong KH, Yun J, et al. Isolation of a regulatory region of activin receptor-like kinase 1 gene sufficient for arterial endothelium-specific expression. Circ Res 2004;94:e72-e77.

13. Gale NW, Yancopoulos GD. Growth factors acting via endothelial cellspecific receptor tyrosine kinases: VEGFs, angiopoietins, and ephrins in vascular development. Genes Dev 1999;13:1055-1066.

14. Wang HU, Chen ZF, Anderson DJ. Molecular distinction and angiogenic interaction between embryonic arteries and veins revealed by ephrinB2 and its receptor Eph-B4. Cell 1998;93:741-753.

15. Torres-Vazquez J, Kamei M, Weinstein BM. Molecular distinction between arteries and veins. Cell Tissue Res 2003;314:43-59.

16. Kokubo H, Miyagawa-Tomita S, Johnson RL. Hesr, a mediator of the Notch signaling, functions in heart and vessel development. Trends Cardiovasc Med 2005;15:190-194.

17. You LR, Lin FJ, Lee CT, et al. Suppression of Notch signalling by the COUP-TFII transcription factor regulates vein identity. Nature 2005;435:98-104.

18. Iso T, Maeno T, Oike $Y$, et al. Dll4-selective Notch signaling induces ephrinB2 gene expression in endothelial cells. Biochem Biophys Res Commun 2006;341:708-714.

19. Rossant J, Cross JC. Placental development: lessons from mouse mutants. Nat Rev Genet 2001;2:538-548.

20. Adamson SL, Lu Y, Whiteley KJ, et al. Interactions between trophoblast cells and the maternal and fetal circulation in the mouse placenta. Dev Biol 2002;250:358-373.

21. Shalaby F, Rossant J, Yamaguchi TP, et al. Failure of blood-island formation and vasculogenesis in Flk-1-deficient mice. Nature 1995;376:62-66.

22. Seki T, Hong KH, Oh SP. Nonoverlapping expression patterns of ALK1 and ALK5 reveal distinct roles of each receptor in vascular development. Lab Invest 2006;86:116-129.

23. Lechleider RJ, Ryan JL, Garrett L, et al. Targeted mutagenesis of Smad1 reveals an essential role in chorioallantoic fusion. Dev Biol 2001;240:157-167.

24. Larsson J, Goumans MJ, Sjostrand L, et al. Abnormal angiogenesis but intact hematopoietic potential in TGF-beta type I receptor-deficient mice. EMBO J 2001;20:1663-1673.

25. Oshima M, Oshima H, Taketo MM. TGF-beta receptor type II deficiency results in defects of yolk sac hematopoiesis and vasculogenesis. Dev Biol 1996;179:297-302.

26. Jadrich JL, O'Connor MB, Coucouvanis E. The TGF beta activated kinase TAK1 regulates vascular development in vivo. Development 2006;133:1529-1541.

27. Yang $X$, Castilla $L H, X u X$, et al. Angiogenesis defects and mesenchymal apoptosis in mice lacking SMAD5. Development 1999;126:1571-1580.

28. Sorensen LK, Brooke BS, Li DY, et al. Loss of distinct arterial and venous boundaries in mice lacking endoglin, a vascular-specific TGFbeta coreceptor. Dev Biol 2003;261:235-250.

29. Adams RH, Porras A, Alonso G, et al. Essential role of p38alpha MAP kinase in placental but not embryonic cardiovascular development. Mol Cell 2000;6:109-116.

30. Hirashima M, Lu Y, Byers $L$, et al. Trophoblast expression of fms-like tyrosine kinase 1 is not required for the establishment of the maternalfetal interface in the mouse placenta. Proc Natl Acad Sci USA 2003;100:15637-15642.

31. Noris M, Perico N, Remuzzi G. Mechanisms of disease: pre-eclampsia. Nat Clin Pract Nephrol 2005;1:98-114.

32. Redman CW, Sargent IL. Latest advances in understanding preeclampsia. Science 2005;308:1592-1594. 
33. Levine RJ, Lam C, Qian C, et al. Soluble endoglin and other circulating antiangiogenic factors in preeclampsia. N Engl J Med 2006;355: 992-1005.

34. Venkatesha S, Toporsian M, Lam C, et al. Soluble endoglin contributes to the pathogenesis of preeclampsia. Nat Med 2006;12:642-649.

35. St Jacques S, Forte M, Lye SJ, et al. Localization of endoglin, a transforming growth factor-beta binding protein, and of CD44 and integrins in placenta during the first trimester of pregnancy. Biol Reprod 1994;51:405-413.
36. Conley BA, Koleva R, Smith JD, et al. Endoglin controls cell migration and composition of focal adhesions: function of the cytosolic domain. J Biol Chem 2004:279:27440-27449.

37. Sanz-Rodriguez F, Guerrero-Esteo M, Botella LM, et al. Endoglin regulates cytoskeletal organization through binding to ZRP-1, a member of the Lim family of proteins. J Biol Chem 2004;279:32858-32868.

38. Jonker L, Arthur HM. Endoglin expression in early development is associated with vasculogenesis and angiogenesis. Mech Dev 2002;110:193-196. 